\title{
The epidemiology of carbapenem-non- susceptible Acinetobacter species in Europe: analysis of EARS-Net data from 2013 to 2017
}

Olaniyi Ayobami ${ }^{1}$, Niklas Willrich ${ }^{1}$, Beneditta Suwono ${ }^{1,2}$, Tim Eckmanns $^{1}$ and Robby Markwart ${ }^{1 *}$ (D)

\begin{abstract}
Background: Due to limited therapeutic options and their association with high mortality and morbidity, carbapenem-non-susceptible Acinetobacter spp. (CNA) are of significant public health importance. This study aimed to describe current epidemiological trends of CNA proportions in Europe and to identify factors that are associated with carbapenem non-susceptibility of isolates from patients with invasive Acinetobacter spp. infections.

Methods: Data from routine carbapenem susceptibility testing of 18,412 invasive clinical Acinetobacter spp. isolates from 30 European countries in 2013-2017 were analysed using descriptive statistical analyses and uni- and multivariable regression analyses. These data were obtained from the European Antimicrobial Resistance Surveillance Network (EARS-Net).

Results: The population-weighted mean proportion of carbapenem-non-susceptible Acinetobacter spp. in Europe is $35.6 \%$ (95\% confidence interval [CI] 29.7-42.0\%). With CNA proportions of $75.5 \%$ (95\% Cl 71.2-79.4\%) and $71.5 \%$ (95\% Cl 66.7-75.9\%) the burden of CNA is particularly high in Southern and Eastern European regions. In contrast, Northern and Western European regions recorded CNA proportions of 2.8\% (95\% Cl 1.2-6.0\%) and 6.3\% (95\% Cl 4.5-8.9\%), respectively. Population-weighted mean CNA proportions are especially high in Acinetobacter spp. isolates from intensive care units (54.0\% [95\% Cl 47.6-60.3\%]). Male gender, age above 20 years and ICU admission were identified as independent factors associated with an increased likelihood of CNA.

Conclusion: The burden of carbapenem-non-susceptible Acinetobacter spp. is particularly high in Southern and Eastern Europe. There is a risk that resistance could spread to other parts of Europe. Therefore, increased efforts in infection control and antibiotic stewardship, particularly in Intensive Care Units, are necessary to combat the spread of CNA in Europe.
\end{abstract}

Keywords: Acinetobacter, Acinetobacter baumannii, Carbapenem resistance, Antimicrobial resistance, Surveillance, EARS-Net

\footnotetext{
* Correspondence: MarkwartR@rki.de

'Department 3: Infectious Disease Epidemiology, Unit 37:

Healthcare-associated Infections, Surveillance of Antibiotic Resistance and Consumption, Robert Koch Institute, Nordufer 20, 13353 Berlin, Germany

Full list of author information is available at the end of the article
}

(c) The Author(s). 2020 Open Access This article is licensed under a Creative Commons Attribution 4.0 International License, which permits use, sharing, adaptation, distribution and reproduction in any medium or format, as long as you give appropriate credit to the original author(s) and the source, provide a link to the Creative Commons licence, and indicate if changes were made. The images or other third party material in this article are included in the article's Creative Commons. licence, unless indicated otherwise in a credit line to the material. If material is not included in the article's Creative Commons licence and your intended use is not permitted by statutory regulation or exceeds the permitted use, you will need to obtain permission directly from the copyright holder. To view a copy of this licence, visit http://creativecommons.org/licenses/by/4.0/ The Creative Commons Public Domain Dedication waiver (http://creativecommons.org/publicdomain/zero/1.0/) applies to the data made available in this article, unless otherwise stated in a credit line to the data. 


\section{Background}

Acinetobacter species spp. are non-fermenting, largely opportunistic, gram negative bacteria that are ubiquitous in the environment. Acinetobacter baumannii complex (Acinetobacter nosocomialis, Acinetobacter pitti and Acinetobacter baumannii) are the most clinically significant among the over 50 species in the Acinetobacter genus [1]. Of all Acinetobacter spp., Acinetobacter baumannii sensu stricto (referred to Acinetobacter baumannii in this article) is responsible for about $90 \%$ of the Acinetobacter spp. clinical infections in humans [1]. Its ability to survive harsh conditions, including desiccation and disinfection, fosters its persistence and spread in hospital environments [2, 3]. The Acinetobacter spp. have also evolved multiple resistance strategies, such as overexpression of efflux pumps, reduced permeability and a diverse array of hydrolytic carbapenemases [4], resulting in resistance to carbapenems and other reserve drugs. In addition, Acinetobacter baumannii is naturally resistant to several antibiotics, including priority "Watch group" antibiotics as defined by the WHO, such as cephalosporin. It has acquired many more resistance strategies, leading to poorer clinical outcomes across many healthcare settings $[5,6]$. Carbapenem-non-susceptible Acinetobacter spp. (CNA) is increasingly recognised as a nosocomial pathogen of significant public health importance worldwide because it presents limited therapeutic options, high treatment costs and is associated with high mortality and morbidity [7-9]. In the World Health Organization's (WHO) recent drug research and development prioritisation exercise for pathogens, CNA emerged as the highest weighted antimicrobial-resistant pathogen [10]. Worldwide, CNA have been implicated in several outbreaks of pneumonia, bloodstream, wound and urinary tract infections, especially among patients with severe morbidities like those on Intensive Care Units (ICU) [11, 12].

According to the 2017 European Antimicrobial Resistance Surveillance Network (EARS-Net) report, proportions of CNA collected from invasive infections are particularly high in many southern and eastern European countries where resistance proportions often exceed 50\% (such as 95\% in Greece, 79\% in Italy, and 53\% in Hungary) [13]. In contrast, western and northern European countries have recorded low proportions of resistance to carbapenems, often less than $5 \%$ (such as $3 \%$ in the United Kingdom, $4 \%$ in Germany and $<1 \%$ Norway, Denmark and Sweden).

Despite these European data, a comprehensive epidemiological picture of invasive carbapenem-non-susceptible Acinetobacter spp. in European hospitals is still lacking. While published EARS-Net reports focus on analyses of national trends, until now it has not been systematically assessed how the likelihood of carbapenem-non-susceptibility is impacted by patients' characteristics (such as gender and age) and other factors (such as hospital unit type, season of the year and antibiotic consumption). This study therefore aims to investigate epidemiological trends of CNA proportions in Europe and seeks to identify factors that are associated with carbapenem-non-susceptibility of isolates from patients with invasive Acinetobacter spp. infections using EARS-Net data from 2013 to 2017.

\section{Methods \\ Study design and the European antimicrobial resistance surveillance database}

We conducted a retrospective observational study on Acinetobacter spp. using data retrieved from the EARS-Net database. EARS-Net is a network of European surveillance systems collecting routine clinical antimicrobial susceptibility (AST) data on invasive isolates (blood and cerebrospinal fluid [CSF]) from the 28 European Union countries as well as Norway and Iceland [13]. Detailed information about the EARS-Net methodology is provided in their surveillance reports and protocols [14]. In EARS-Net, no information on the specific species of the Acinetobacter spp. isolates are reported. Acinetobacter spp. are classified by EARS-Net as sensitive (S), intermediate (I), or resistant (R) to antimicrobial drugs, based on the standards used in the participating laboratories, such as the guidelines of the European Committee on Antimicrobial Susceptibility Testing (EUCAST), Clinical and Laboratory Standards Institute (CLSI) or other national guidelines. In our data set, more than $98 \%$ of all isolates with information on the used guideline have their AST interpreted with EUCAST or CLSI. As reported by the ECDC, there is a general consensus between the EUCAST and CLSI interpretation for carbapenem susceptibility testing for Acinetobacter spp. [15].

In addition to S-I-R data, individual laboratories provide further epidemiological information. This includes the date of specimen collection, specimen type (i. e. blood and CSF), care type (such as inpatient or outpatient care), patient gender and patient age as well as the hospital unit (such as ICU or internal medicine unit), where the sample was collected.

\section{Selection of Acinetobacter spp. isolates}

We extracted data for Acinetobacter spp. from the TESSy database in February 2019 with the approval of the European Centre for Disease Prevention and Control. Only the first isolate from a given patient in the respective year is included in the TESSy database of EARS-Net. We excluded isolates with multiple AST tests against the same antibiotic as well as isolates with the same unique identification number. In the next steps, we excluded isolates from outpatient care and isolates that were neither tested against meropenem nor imipenem. Since the initial pilot study of EARS-Net was conducted 
in 2012 and included data from only 18 countries, we exclusively analysed data on Acinetobacter spp. from 2013 to 2017, when all 30 countries provided data to EARS-Net.

\section{Outcomes and variables}

The primary outcome of interest is the populationweighted proportion of carbapenem-non-susceptible Acinetobacter spp. isolates among all Acinetobacter spp. isolates, expressed as a percentage (\%) and with 95\% confidence intervals (95\% CI). An Acinetobacter spp. isolate was defined as carbapenem-non-susceptible if it was tested resistant or intermediate against meropenem and/ or imipenem. Clinical specimens were grouped by sampling site into either blood or cerebrospinal fluid. Patient ages were categorized into six age categories $(<1,1-19$, $20-39,40-59,60-79$ and $\geq 80$ years). Patient genders were classified as female or male. The isolates' country of origin were grouped into four major European regions (Northern: Denmark, Finland, Iceland, Ireland, Norway, Sweden, United Kingdom; Western: Austria, Belgium, France, Germany, Luxembourg, Netherlands; Southern: Croatia, Cyprus, Greece, Hungary, Italy, Malta, Portugal, Slovenia, Spain; Eastern: Bulgaria, Czech Republic, Estonia, Latvia, Lithuania, Poland, Romania, Slovakia). Hospital units were categorised into "ICU", "Internal medicine", "Surgery", "Oncology" and "Others". To determine potential seasonal trends in the occurrence of CNA, the months in which the sample were collected were categorised into "Warm months" (May - September) and "Cold months" (October - April). In order to investigate the populationweighted proportion of co-resistance to gentamicin and ciprofloxacin among CNA isolates, only isolates that were separately tested against gentamicin and ciprofloxacin were selected. An Acinetobacter spp. isolate was defined as ciprofloxacin- or gentamicin-non-susceptible if it was tested intermediate or resistant against ciprofloxacin and gentamicin, respectively.

\section{Statistical analyses}

All statistical analyses were performed using $\mathrm{R}$ version 3.5.1 [16] and the "survey" package (version 3.35). Proportions and 95\% confidence intervals of carbapenemnon-susceptibility were estimated accounting for clustering at the hospital level and stratified by country level. For all calculations in all strata, country populationbased weighting was applied. The population data were obtained from the Eurostat database (https://ec.europa. eu/eurostat/data/database). Weightings were used for each country's population in the calculation of nonsusceptibility proportions in order to ensure that the data contributed proportionally to their population sizes. This was done to minimise biases from significant differences in isolate numbers between the countries. The potential association between different variables and carbapenem-non-susceptibility was analysed using univariable and multivariable logistic regression analyses accounting for clustering and using weights proportional to each country's population sizes as described above. For the univariable analyses, the following predictors for carbapenem-non-susceptibility were considered: Year of sampling, gender, age group, specimen type, European region, hospital unit type and season. These variables were selected before conducting the analysis and were based on the availability of the data and our prior hypotheses on which variables may be associated with carbapenem-non-susceptibility in Acinetobacter spp. We included all variables from the univariable analyses in the model for the multivariable analysis.

\section{Results}

\section{Dataset characteristics}

The baseline characteristics of the analysed Acinetobacter spp. isolates are outlined in Table 1. In total, 18,412 isolates of Acinetobacter spp. from 18,167 patients were collected in 1191 hospitals across Europe between 2013 and 2017. The majority of isolates originated from elderly patients (median age: 64 years). For the isolates with a reported patient gender $(n=12,678)$, the female/male ratio was 0.72 . The clinical samples were predominantly from blood specimens (97\%). Isolates were most frequently collected from patients treated on ICUs (41\%) and internal medicine units (25\%), followed by surgical units (11\%) and oncology units (5\%). About threequarters of the isolates (73\%) were from the Southern and Eastern regions of Europe, which represented 45\% of the mean population of the 30 countries included in the study.

\section{Proportion and temporal trend of CNA in Europe}

Between 2013 and 2017, the population-weighted mean proportion of carbapenem-non-susceptible Acinetobacter spp. isolates in Europe was 35.6\% (95\% CI 29.7-42.0\%). However, when disaggregated into regions, the profound Southern-Eastern to Northern-Western gradient previously described in EARS-Net reports [13, 17] became apparent. While the Southern and Eastern regions reported CNA proportions of $75.5 \%$ (95\% CI 71.2-79.4\%) and $71.5 \%$ (95\% CI 66.7-75.9\%), respectively, the Northern and Western regions reported CNA proportions as low as $2.8 \%$ (95\% CI 1.2-6.0\%) and 6.3\% (95\% CI 4.5$8.9 \%$ ), respectively (Additional File 1: Additional Table 1). Univariable and multivariable regression analyses confirmed that Acinetobacter spp. isolates from Southern and Eastern Europe were more likely to be carbapenem-non-susceptible than isolates from Northern and Western European regions (Table 2). 
Table 1 Baseline characteristics of analysed invasive Acinetobacter spp. isolates

\begin{tabular}{|c|c|c|c|c|c|c|c|c|c|c|}
\hline \multirow{2}{*}{ Number of isolates } & \multicolumn{2}{|c|}{ Europe (total) } & \multicolumn{2}{|c|}{ Northern region } & \multicolumn{2}{|c|}{ Western region } & \multicolumn{2}{|c|}{ Southern region } & \multicolumn{2}{|c|}{ Eastern region } \\
\hline & 18,412 & & 610 & & 4367 & & 10,522 & & 2913 & \\
\hline \multicolumn{11}{|l|}{ Year of sampling } \\
\hline $2013(n, \%)$ & 3110 & 16.89 & 202 & 33.11 & 637 & 14.59 & 2032 & 19.31 & 239 & 8.20 \\
\hline $2014(n, \%)$ & 3363 & 18.27 & 105 & 17.21 & 706 & 16.17 & 2074 & 19.71 & 478 & 16.41 \\
\hline 2015 (n, \%) & 4037 & 21.93 & 105 & 17.21 & 851 & 19.49 & 2386 & 22.68 & 695 & 23.86 \\
\hline $2016(n, \%)$ & 3789 & 20.58 & 72 & 11.80 & 1033 & 23.65 & 1876 & 17.83 & 808 & 27.74 \\
\hline $2017(n, \%)$ & 4113 & 22.34 & 126 & 20.66 & 1140 & 26.10 & 2154 & 20.47 & 693 & 23.79 \\
\hline \multicolumn{11}{|l|}{ Patient gender } \\
\hline Female (n, \%) & 5317 & 28.88 & 300 & 49.18 & 1845 & 42.25 & 2122 & 20.17 & 1050 & 36.05 \\
\hline Male (n, \%) & 7361 & 39.98 & 309 & 50.66 & 2295 & 52.55 & 3184 & 30.26 & 1573 & 54.00 \\
\hline NA $(n, \%)$ & 5734 & 31.14 & 1 & 0.16 & 227 & 5.20 & 5216 & 49.57 & 290 & 9.96 \\
\hline Sex ratio $(f / m)$ & 0.72 & & 0.97 & & 0.80 & & 0.67 & & 0.67 & \\
\hline \multicolumn{11}{|l|}{ Patient age } \\
\hline$<1$ years $(\mathrm{n}, \%)$ & 503 & 2.73 & 31 & 5.08 & 116 & 2.66 & 281 & 2.67 & 75 & 2.57 \\
\hline $1-19$ years $(n, \%)$ & 659 & 3.58 & 71 & 11.64 & 239 & 5.47 & 230 & 2.19 & 119 & 4.09 \\
\hline 20-39years (n, \%) & 1136 & 6.17 & 66 & 10.82 & 365 & 8.36 & 399 & 3.79 & 306 & 10.50 \\
\hline 40-59years (n, \%) & 2956 & 16.05 & 104 & 17.05 & 874 & 20.01 & 1231 & 11.70 & 747 & 25.64 \\
\hline 60-79years $(n, \%)$ & 5743 & 31.19 & 218 & 35.74 & 1746 & 39.98 & 2499 & 23.75 & 1280 & 43.94 \\
\hline $80+$ years $(n, \%)$ & 2243 & 12.18 & 120 & 19.67 & 853 & 19.53 & 911 & 8.66 & 359 & 12.32 \\
\hline NA $(n, \%)$ & 5172 & 28.09 & 0 & 0.00 & 174 & 3.98 & 4971 & 47.24 & 27 & 0.93 \\
\hline Age (median, IQR) & 64.0 & $49-76$ & 62.0 & $36-76$ & 66.0 & $51-78$ & 65.0 & $50-76$ & 63 & $48-73$ \\
\hline \multicolumn{11}{|l|}{ Specimen (sampling site) } \\
\hline Blood (n, \%) & 17,818 & 96.77 & 591 & 96.89 & 4304 & 98.56 & 10,108 & 96.07 & 2815 & 96.64 \\
\hline $\operatorname{CSF}(n, \%)$ & 594 & 3.23 & 19 & 3.11 & 63 & 1.44 & 414 & 3.93 & 98 & 3.36 \\
\hline \multicolumn{11}{|l|}{ Hospital unit type } \\
\hline Intensive care & 7605 & 41.30 & 36 & 5.90 & 702 & 16.08 & 5352 & 50.86 & 1515 & 52.01 \\
\hline Internal medicine & 4612 & 25.05 & 154 & 25.25 & 1452 & 33.25 & 2509 & 23.85 & 497 & 17.06 \\
\hline Surgical unit & 1936 & 10.51 & 71 & 11.64 & 415 & 9.50 & 1204 & 11.44 & 246 & 8.44 \\
\hline Oncology & 919 & 4.99 & 46 & 7.54 & 458 & 10.49 & 267 & 2.54 & 148 & 5.08 \\
\hline Other (n, \%) & 2554 & 13.87 & 155 & 25.41 & 1042 & 23.86 & 908 & 8.63 & 449 & 15.41 \\
\hline NA (n, \%) & 786 & 4.27 & 148 & 24.26 & 298 & 6.82 & 282 & 2.68 & 58 & 1.99 \\
\hline Patients with Acinetobacter spp. isolates ( $n$ ) & 18,167 & & 603 & & 4249 & & 10,415 & & 2905 & \\
\hline Hospitals (n) & 1191 & & 139 & & 568 & & 366 & & 118 & \\
\hline
\end{tabular}

Abbreviations: 95\% Cl 95\% confidence interval, CSF Cerebrospinal fluid, IQR Interquartile range, NA Not available

For Europe as a whole, the proportion of Acinetobacter spp. isolates non-susceptible against carbapenems showed an initial apparent increase between 2013 (32.9\% [95\% CI 25.8-40.7\%]) and 2015 (39.8\% [95\% CI 32.646.0\%]), followed by a decline until 2017 (36.0 [95\% CI 28.7-44.0\%) (Fig. 1 and Additional File 1: Additional Table 1). Subregional analyses showed that CNA proportions remained stable in Northern, Western and Southern European regions. In contrast, CNA proportions increased in Eastern Europe from 49.7\% (95\% CI $41.0-58.5 \%)$ in 2013 to 72.6 (95\% CI $67.1-77.4 \%$ ) in
2015 and remained stable in 2016 and 2017 (Fig. 1 and Additional File 1: Additional Table 1).

\section{Age and gender}

In order to understand the possible influence of the age and gender of patients on CNA infections, these two factors were tested for any association with carbapenemnon-susceptibility. The population-weighted mean proportions for the whole of Europe show that isolates from infants and babies ( $0-1$ years) as well as from children and adolescents (1-20 years) exhibited markedly lower 
Table 2 Analysis of factors associated with carbapenem-non-susceptibility in invasive Acinetobacter spp. isolates

\begin{tabular}{|c|c|c|c|c|c|c|}
\hline & \multicolumn{3}{|c|}{$\underline{\text { Univariable analysis }}$} & \multicolumn{3}{|c|}{ Multivariable analysis } \\
\hline & $\mathrm{OR}$ & $(95 \% \mathrm{Cl})$ & $p$-value & $\mathrm{OR}$ & $(95 \% \mathrm{Cl})$ & $\mathrm{p}$-value \\
\hline \multicolumn{7}{|l|}{ Year of sampling } \\
\hline 2013-2017 & 1.02 & $(0.88-1.18)$ & 0.844 & 1.03 & $(0.94-1.13)$ & 0.571 \\
\hline \multicolumn{7}{|l|}{ European region } \\
\hline Northern & 1 & - & - & 1 & - & - \\
\hline Western & 2.37 & $(0.98-5.74)$ & 0.056 & 2.66 & $(1.04-6.82)$ & 0.041 \\
\hline Southern & 109 & $(47.2-250)$ & $<0.001$ & 83.76 & (33.79-207.66) & $<0.001$ \\
\hline Eastern & 88.4 & (38.3-204) & $<0.001$ & 75.87 & $(31.23-184.32)$ & $<0.001$ \\
\hline \multicolumn{7}{|l|}{ Patient gender } \\
\hline Female & 1 & - & - & 1 & - & - \\
\hline Male & 1.54 & $(1.36-1.73)$ & $<0.001$ & 1.17 & $(1.03-1.33)$ & 0.013 \\
\hline \multicolumn{7}{|l|}{ Patient age } \\
\hline$<1$ years & 0.36 & $(0.16-0.82)$ & 0.014 & 0.24 & $(0.10-0.59)$ & 0.002 \\
\hline $1-19$ years & 0.20 & $(0.12-0.32)$ & $<0.001$ & 0.28 & $(0.16-0.49)$ & $<0.001$ \\
\hline 20-39 years & 1 & - & - & 1 & - & - \\
\hline 40-59 years & 1.36 & $(1.01-1.84)$ & 0.044 & 1.25 & $(0.88-1.77)$ & 0.217 \\
\hline $60-79$ years & 1.20 & $(0.91-1.58)$ & 0.195 & 1.21 & $(0.92-1.56)$ & 0.178 \\
\hline $80+$ years & 0.78 & $(0.58-1.07)$ & 0.123 & 1.16 & $(0.85-1.59)$ & 0.352 \\
\hline \multicolumn{7}{|c|}{ Specimen (sampling site) } \\
\hline Blood & 1 & - & - & 1 & - & - \\
\hline CSF & 1.42 & $(1.00-2.01)$ & 0.052 & 0.78 & $(0.45-1.35)$ & 0.373 \\
\hline \multicolumn{7}{|l|}{ Hospital unit type } \\
\hline Internal medicine & 1 & - & - & 1 & - & - \\
\hline Intensive care & 6.62 & $(5.26-8.33)$ & $<0.001$ & 3.79 & $(2.94-4.88)$ & $<0.001$ \\
\hline Oncology & 0.49 & $(0.32-0.74)$ & $<0.001$ & 0.61 & $(0.43-0.87)$ & 0.006 \\
\hline Surgery & 1.47 & $(1.16-1.88)$ & 0.002 & 1.17 & $(0.85-1.60)$ & 0.341 \\
\hline Other & 0.64 & $(0.47-0.85)$ & 0.003 & 0.81 & $(0.62-1.06)$ & 0.13 \\
\hline \multicolumn{7}{|l|}{ Season } \\
\hline Warm months & 0.83 & $(0.73-0.94)$ & 0.003 & 0.86 & $(0.73-1.02)$ & 0.087 \\
\hline Cold months & 1 & - & - & 1 & - & - \\
\hline
\end{tabular}

Abbreviations: 95\% Cl 95\% confidence interval, CSF Cerebrospinal fluid, OR Odds ratio

Carbapenem-non-susceptible Acinetobacter spp.

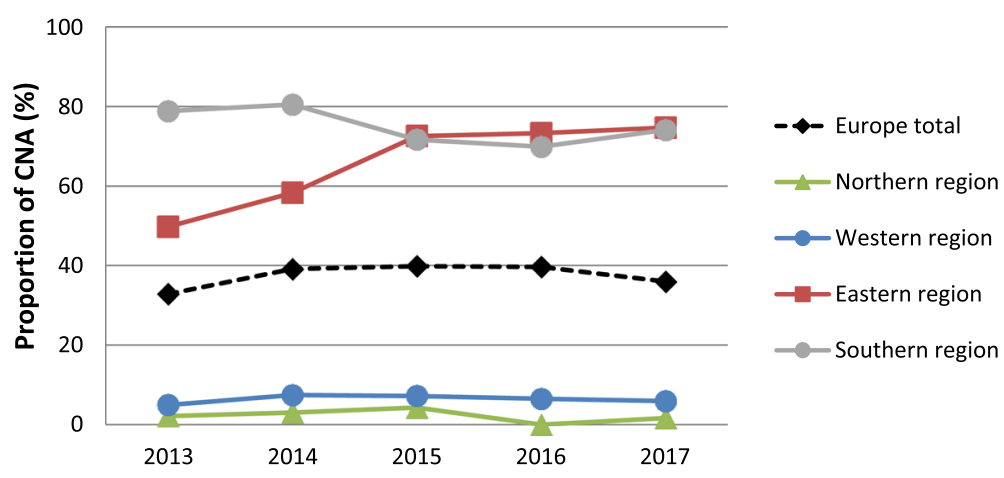

Fig. 1 Time trend of carbapenem-non-susceptible Acinetobacter spp. Time trend of carbapenem-non-susceptible Acinetobacter spp. (CNA) as population-weighted mean proportions (\%) among all Acinetobacter spp. isolates, with corresponding $95 \%$ confidence intervals for the whole of Europe and European regions 
CNA proportions (15.8\% [95\% CI 9.3-25.5\%] and 16.2\% [95\% CI 11.9-21.8], respectively) compared to older age groups, where $>30 \%$ of all Acinetobacter spp. isolates were carbapenem-non-susceptible (Fig. 2 and Additional File 1: Additional Table 1). This finding is supported by univariable and multivariable analyses, which confirmed that Acinetobacter spp. isolates from patients aged 0-1 years and 1-20 years are less likely to be carbapenemnon-susceptible than isolates from older patients (Table 2). Importantly, similar patterns of relative age differences were observed in Western, Eastern and Southern European regions (Additional File 1: Additional Table 1), irrespective of the overall mean CNA proportion reported in these regions. There was an overall population-weighted mean difference of $3 \%$ in the proportion of CNA among isolates between female (32.8\% [95\% CI 26.9-39.2\%]) and male (35.9\% [95\% CI 29.742.6\%]) patients (Additional File 1: Additional Table 1). Univariable and multivariable analyses show that male patients have a slightly higher likelihood of CNA than female patients (Table 2).

\section{Clinical specimen}

Analysis by specimen (sampling site) indicates a higher carbapenem-non-susceptibility proportion in CSF isolates $(41.0 \%$ [95\% CI 35.1-47.2\%]) than observed in blood isolates (35.6\% [95\% CI 29.6-42.0\%]) (Additional File 1: Additional Table 1). However, only $3 \%$ of the isolates were from CSF, and no statistically significant association between specimen type and the likelihood of CNA infection was found in univariable and multivariable regression analyses (Table 2).

\section{Hospital unit type}

Analyses of various hospital unit types where the samples were collected reveal substantial differences in CNA proportions (Fig. 3 and Additional File 1: Additional Table 1). For the whole population-weighted European

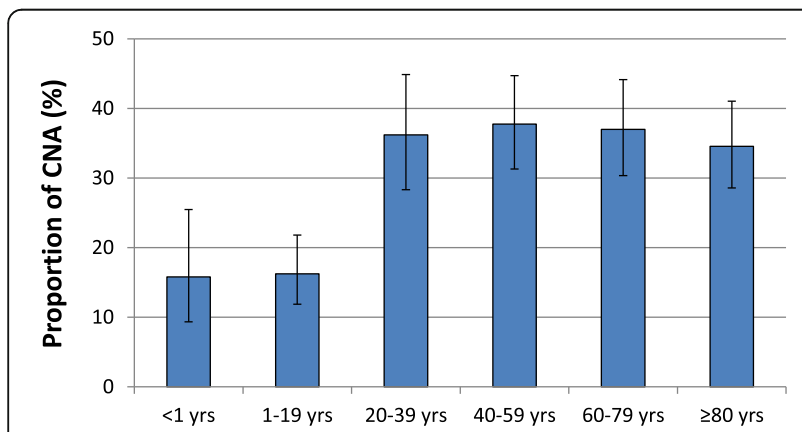

Fig. 2 Carbapenem-non-susceptible Acinetobacter spp. stratified into age. Carbapenem-non-susceptible Acinetobacter spp. (CNA) as a proportion (\%) of all Acinetobacter spp. isolates, with corresponding 95\% confidence intervals for age categories. Yrs: Years

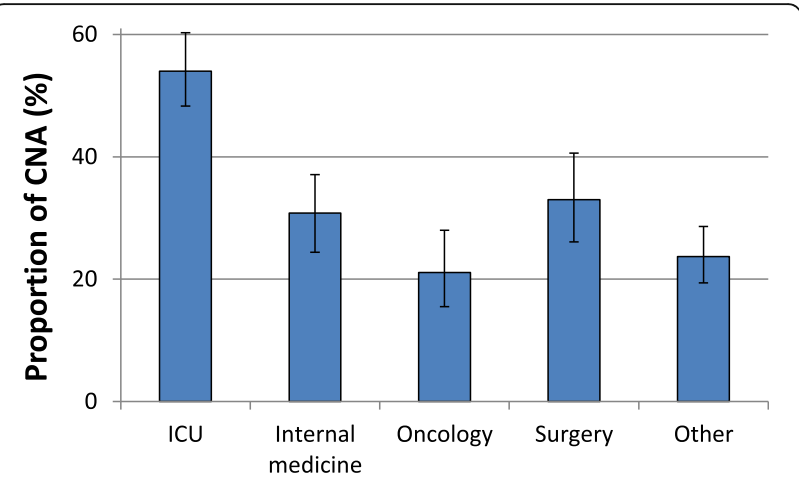

Fig. 3 Carbapenem-non-susceptible Acinetobacter spp. stratified by hospital unit types. Carbapenem-non-susceptible Acinetobacter spp. (CNA) as population-weighted mean proportions (\%) among all Acinetobacter spp. isolates, with corresponding 95\% confidence intervals for hospital unit types. ICU: Intensive care unit

sample set, ICUs recorded a significantly higher proportion of CNA (54.0\% [95\% CI 47.6-60.3\%]) compared to other hospital units. Internal medicine units and surgical wards recorded CNA proportions of $30.8 \%$ (95\% CI 25.1-37.1\%) and 33.0 (95\% CI 26.1-40.6\%), respectively. Univariable and multivariable regression analyses confirmed that the likelihood of CNA on ICUs is markedly more likely than on other hospital units (Table 2). The likelihood of CNA acquisition on oncological units is lower than on internal medicine units (OR: 0.61 [95\% CI $0.43-0.87, p=0.006]$. Substantially higher CNA proportions of invasive Acinetobacter spp. isolates collected from ICUs in relation to other hospital units were observed across all European regions (Additional File 1: Additional Table 1).

\section{Seasonality}

For Europe as a whole, CNA proportions did not differ between samples that were collected during the "Cold months" (36.4\% [95\% CI 30.4-43.0\%] and "Warm months" (34.6\% [95\% CI 28.7-41.0\%]) (Additional File 1: Additional Table 1). No statistically significant association between seasonality and CNA proportions was therefore found in multivariable analyses (OR: 0.86 [95\% CI 0.73-1.02], $p=0.087$ ) (Table 2). Moreover, there were no major differences in CNA proportions between cold and warm months on European regional level (Northern: $2.8 \%$ [95\% CI $0.9-7.9 \%$ ] vs. $2.8 \%$ [95\% CI $0.9-8.6 \%$; Western: 6.4\% [95\% CI 4.5-9.0\%] vs. 6.4\% [95\% CI 4.49.1\%]; Southern: $77.3 \%$ [95\% CI $72.6-81.4 \%$ ] vs. $73.3 \%$ [95\% CI 60.5-77.7\%]; Eastern: 73.7\% [95\% CI 68.178.6\%] vs. $68.9 \%$ [95\% CI $64.2-73.2 \%]$.

\section{Co-resistance}

Since fluoroquinolones (e.g. ciprofloxacin) and aminoglycoside antibiotics (e.g. gentamicin) represent potential 
therapy options to treat CNA infections, ciprofloxacin and gentamicin resistance proportions among carbapenemsusceptible and carbapenem-non-susceptible Acinetobacter spp. isolates were studied. The majority of Acinetobacter spp. isolates that are non-susceptible to carbapenems also exhibited non-susceptibility to ciprofloxacin $(65.1 \%$ [95\% CI 56.7-72.6\%]) and gentamicin (81.0\% [95\% CI 77.1-84.4\%]). In contrast, among carbapenem-susceptible Acinetobacter strains, ciprofloxacin- and gentamicin nonsusceptibility proportions were $3.4 \%$ [95\% CI $2.6-4.4 \%$ ] and $14.7 \%$ [95\% CI 10.9-19.5\%]), respectively. Importantly, co-resistance to ciprofloxacin among CNA is particularly high in Southern and Eastern European regions (95.0\% [95\% CI 93.5-96.2\%]) and 87.0\% (95\% CI 82.190.7\%], respectively. Similarly, both regions also exhibit a high burden of gentamicin co-non-susceptibility among carbapenem-non-susceptible Acinetobacter isolates (Southern: 96.1\% [95\% CI 94.6-97.2\%], Eastern: 89.4\% [95\% CI 86.6-91.6\%]).

\section{Discussion}

In this study, we assessed epidemiological trends of invasive carbapenem-non-susceptible Acinetobacter spp. isolates from hospital patients in the European Union and the European Economic Area (EAA) using data from EARS-Net. Similar to the conclusions reached previously by the ECDC [1], our study demonstrated the persisting challenge of CNA in Europe as the country-population weighted mean proportion of non-susceptible isolates exceeds 30\% for the period between 2013 and 2017. The CNA proportion observed in our study is similar to the CNA proportion (35\%) reported in a recently published nationwide survey done in the United States among hospitalized patients [18]. A recent global study showed that carbapenem-non-susceptibility in Acinetobacter baumannii complex isolates from both invasive and noninvasive infections was considerably higher in the AsiaPacific region ( 79\%), Latin America ( 85\%) and North America ( 45\%) compared to the mean CNA proportion in Europe described in our study [19]. Importantly, in Europe the Southern-Eastern to Northern-Western gradient persists. In the Southern and Eastern regions, more than $70 \%$ of all Acinetobacter spp. isolates from invasive infections were carbapenem-non-susceptible, while in the North and the West, CNA proportions of less than 10\% were observed. This gradient seen in the non-susceptibility of invasive Acinetobacter spp. to carbapenems was also observed for other pathogens in Europe, including Pseudomonas aeruginosa and Klebsiella pneumoniae [13], suggesting a systematic higher burden of invasive infections with drug-resistant Gramnegative pathogens in Southern and Eastern European regions. High CNA proportions in the Southern and Eastern regions is of great clinical concern, particularly in light of growing resistance against other antimicrobial drugs used as alternative therapeutic options to treat CNAs infections, such as fluoroquinolones and aminoglycoside antibiotics. Our analyses show that on average, $>70 \%$ of CNA are also non-susceptible to ciprofloxacin or gentamicin in Southern and Eastern Europe.

Differences in antimicrobial use, infection control and health care utilisation practices between the countries are possible reasons for the variation in the reported proportion of drug-resistant Acinetobacter spp. isolates [20]. Previous studies have shown limited organizational support and practice of infection control measures like standard precautions among health workers that work in the medical ward as well as in critical areas like the ICU in Eastern (e.g. Poland) and Southern European (e.g. Greece) countries [21-23]. The 2017 ESAC-Net report showed an increasing trend of carbapenem consumption between 2013 and 2017 in many of the Southern and Eastern region countries, including Latvia, Lithuania, Romania, Poland and Hungary [24]. This might partly explain the surge in carbapenem-non-susceptibility seen in these regions. In fact, an association between hospital carbapenem consumption and carbapenem-resistance rates of Acinetobacter have been described by multiples studies [25-29]. Beside antibiotic usage, other possible explanations for the observed gradient in CNA proportion have been suggested, such as differences in climate and socio-economic conditions across these European regions [30, 31].

In addition to regional differences in CNA proportions in Europe, this study identified factors that are associated with a higher likelihood of acquisition of carbapenemnon-susceptible Acinetobacter spp. Adult patients $(>20$ years) show markedly higher CNA proportions than both babies and infants ( $<1$ years) and children and adolescents between 1 and 20 years. Importantly, the differences in non-susceptibility proportion between adults and babies/ adolescents is also observed in regional analyses in Western, Southern and Eastern European regions, suggesting that the higher proportion of CNA in adult patients is a common feature in invasive Acinetobacter spp. infections, independent of inter-regional differences in CNA proportions. This finding is consistent with other studies that found children and adolescents are less susceptible to several infections with drug-resistant bacteria, including carbapenem-non-susceptible Klebsiella pneumoniae [32], Pseudomonas aeruginosa [33], as well as methicillinresistant Staphylococcus aureus [34] and vancomycinresistant Enterococcus faecium [35, 36]. Several factors may explain these age differences. It has been shown that elderly patients are more likely to be colonised with drugresistant pathogens due to more frequent exposure to antibiotics throughout their lives. Moreover, elderly patients have more comorbidities than younger patients and are 
more likely to reside in nursing homes or other healthcare facilities, both factors that have been shown to be associated with increased AMR [37]. This study also shows that male patients are associated with an increased likelihood of CNA compared to female patients. Male gender is also associated with higher proportions of other carbapenemnon-susceptible pathogens, including of Klebsiella pneumoniae and other Gram-negative rods [32, 38].

Unsurprisingly, most of the isolates in this study are from the ICUs, which is a known hotbed for nosocomial transmission of Acinetobacter spp. [11, 39]. This study shows that the proportion of CNA is highest among isolates collected in the ICU where many patients tend to have severe comorbidities and are exposed to antibiotics and devices that enable the transmission of pathogens [40-42]. Studies have also reported that admission to the ICU is a risk factor for the acquisition and isolation of CNA infections in other regions of the world, such as Northern Africa [43, 44].

\section{Strengths and limitations}

To our knowledge, this study is the largest and most comprehensive analysis of CNA infections in Europe, with more than 18,000 clinical isolates from 30 countries in the EU/EAA. EARS-Net data are based on routine clinical antimicrobial susceptibility data from national surveillance programmes. The accuracy and validity of the AMR data has been assured through annual external quality assessments on all laboratories from all countries, which showed they all performed generally well [45]. However, it is important to consider the limitations of EARS-Net data. Firstly, participating national laboratories and hospitals might not be representative of an individual country, although according to the EARS-Net report 2017 [13], many countries across all regions reached a high isolate sample representativeness in their national surveillance systems. Secondly, population coverage varied among reporting countries. To address this issue, all statistical analyses used weightings based on the population sizes of the individual countries similar to that done in the EARS-Net reports. Thirdly, Acinetobacter spp. are not identified to the species level in the EARS-Net database. This is important in terms of carbapenem resistance, as $A$. baumannii isolates are more frequently carbapenem-resistant that other Acinetobacter species. Therefore, the differential proportion of $A$. baumannii among all Acinetobacter species across countries and regions might impact the CNA proportions recorded in EARS-Net. Last, different sampling routines in different healthcare settings such as due to limited resources can result in biased estimates of CNA proportions across European regions. However, due its clinical significance, inclusion of only invasive clinical specimen limits the bias that may result from some of the inconsistencies in sampling, although frequency of blood sampling varies between hospitals and countries.

\section{Conclusion}

Carbapenem-non-susceptible Acinetobacter spp. pose a significant threat to public health in Europe, particularly in the Southern and Eastern regions where more than $70 \%$ of all invasive Acinetobacter isolates were non-susceptible to carbapenems. The proportion of CNA is particularly high on ICUs and among older patients. These findings raise concern for inter-regional spread to areas with traditionally low CNA proportions from contiguous European countries with high CNA proportions. In order to prevent the spread of CNA infections, continued surveillance and implementation of effective infection prevention and control measures are needed, particularly in high-risk settings and through targeting high risk groups.

\section{Supplementary information}

Supplementary information accompanies this paper at https://doi.org/10. 1186/s13756-020-00750-5.

Additional file 1: Additional Table 1. Proportions (\%) and 95\% confidence intervals of carbapenem-non-susceptible Acinetobacter spp. isolates.

\section{Abbreviations}

AMR: Antimicrobial Resistance; AST: Antimicrobial susceptibility testing; Cl: Confidence intervals; CLSI: Clinical \& Laboratory Standards Institute; CNA: Carbapenem-nonsusceptible Acinetobacter spp.; CSF: Cerebrospinal fluid; EARS-Net: European Antimicrobial Resistance Surveillance Network; ECDC: European Centre for Disease Prevention and Control; EUCAST: European Committee on Antimicrobial Susceptibility Testing; ICU: Intensive care unit; IQR: Interquartile range; OR: Odds ratio; WHO: World Health Organization

\section{Acknowledgements}

We would like to thank all EARS-Net participating laboratories and hospitals in the EU/EEA countries for providing data. We wish to thank the European Centre for Disease Prevention and Control for providing the dataset for our paper. We thank our colleagues Angelina Taylor and Sebastian Haller at the Robert Koch Institute for their contribution to this study.

\section{Disclaimer}

The views and opinions of the authors expressed herein do not necessarily state or reflect those of the ECDC. The accuracy of the authors' statistical analysis and the findings they report are not the responsibility of the ECDC. The ECDC is not responsible for conclusions or opinions drawn from the data provided. The ECDC is not responsible for the correctness of the data and for data management, data merging and data collation after provision of the data. The ECDC shall not be held liable for improper or incorrect use of the data.

\section{Authors' contributions}

RM and OA designed the study, performed analyses and wrote the manuscript. NW: performed analyses. BS, TE and RM conceptualised the study. All authors critically reviewed and edited the final manuscript. The author(s) read and approved the final manuscript.

\section{Funding}

The analysis was conducted by internal funds of the Robert Koch Institute as a Federal Institute within the portfolio of the Ministry of Health.

Availability of data and materials

Raw data were obtained from the TESSy database (EARS-Net) with the approval of the ECDC. All raw data can be provided on reasonable request with the approval of the ECDC. 


\section{Ethics approval and consent to participate}

The study only includes anonymised routine surveillance data. Ethical approval for analysis of such surveillance data is not required according to the Medical Association's professional code of conduct.

\section{Consent for publication}

All authors read and approved the final manuscript and gave consent for publication.

\section{Competing interests}

The authors declare that they have no competing interests.

\section{Author details}

Department 3: Infectious Disease Epidemiology, Unit 37:

Healthcare-associated Infections, Surveillance of Antibiotic Resistance and Consumption, Robert Koch Institute, Nordufer 20, 13353 Berlin, Germany. ${ }^{2}$ Federal Institute for Risk Assessment, Unit Epidemiology, Zoonoses and Antimicrobial Resistance Department Biology Safety, Berlin, Germany.

\section{Received: 23 December 2019 Accepted: 4 June 2020}

\section{Published online: 19 June 2020}

\section{References}

1. ECDC. Carbapenem-resistant Acinetobacter baumanni in healthcare settings. Stockholm: European Centre for Disease Prevention and Control; 2016.

2. Abdulzahra AT, Khalil MAF, Elkhatib WF. First report of colistin resistance among carbapenem-resistant Acinetobacter baumannii isolates recovered from hospitalized patients in Egypt. New Microbes New Infect. 2018;26:53-8.

3. Fournier PE, Richet $\mathrm{H}$. The epidemiology and control of Acinetobacter baumannii in health care facilities. Clin Infect Dis. 2006;42(5):692-9.

4. Karah $\mathrm{N}$, et al. Insights into the global molecular epidemiology of carbapenem non-susceptible clones of Acinetobacter baumannii. Drug Resist Updat. 2012;15(4):237-47.

5. Munoz-Price LS, Weinstein RA. Acinetobacter infection. N Engl J Med. 2008; 358(12):1271-81

6. Sharland $\mathrm{M}$, et al. Classifying antibiotics in the $\mathrm{WHO}$ essential medicines list for optimal use-be AWaRe. Lancet Infect Dis. 2018;18(1):18-20.

7. Kempf M, Rolain JM. Emergence of resistance to carbapenems in Acinetobacter baumannii in Europe: clinical impact and therapeutic options. Int J Antimicrob Agents. 2012;39(2):105-14.

8. Higgins $P G$, et al. Global spread of carbapenem-resistant Acinetobacter baumannii. J Antimicrob Chemother. 2010;65(2):233-8.

9. Zhen $X$, et al. The difference in medical costs between carbapenem-resistant Acinetobacter baumannii and non-resistant groups: a case study from a hospital in Zhejiang province, China. Eur J Clin Microbiol Infect Dis. 2017;36(10):1989-94.

10. Tacconelli $\mathrm{E}$, et al. Discovery, research, and development of new antibiotics: the WHO priority list of antibiotic-resistant bacteria and tuberculosis. Lancet Infect Dis. 2018:18(3):318-27.

11. Kim UJ, et al. Update on the epidemiology, treatment, and outcomes of Carbapenem-resistant Acinetobacter infections. Chonnam Med J. 2014:50(2):37-44.

12. Kohlenberg A, et al. Outbreak of carbapenem-resistant Acinetobacter baumannii carrying the carbapenemase OXA-23 in a German university medical Centre. J Med Microbiol. 2009:58(Pt 11):1499-507.

13. ECDC. Surveillance of Antimicrobial Resistance in Europe. In: Surveillance Report. Stockholm: European Centre for Disease Prevention and Control 2017.

14. ECDC, Antimicrobial resistance: Tessy reporting protocol (2018). 2018.

15. European Centre for Disease Prevention and Control. In: E.C.f.D.P.a. Control, editor. Rapid Risk Assessment: Carbapenem-resistant Acinetobacter baumannii in healthcare settings, in Rapid Risk Assessment. Stockholm: European Centre for Disease Prevention and Control; 2016.

16. Team, R.C., R: A language and environment for statistical computing. R Foundation for Statistical Computing. Available online at https://umw.R-project.org/. 2018

17. ECDC. Antimicrobial resistance surveillance in Europe. In: Annual Report of the European Antimicrobial Resistance Surveillance Network (EARS-Net) 2015 Surveillance Report; 2017.

18. Gupta $V$, et al. National prevalence estimates for resistant Enterobacteriaceae and Acinetobacter species in hospitalized patients in the United States. Int J Infect Dis. 2019;85:203-11.

19. Gales AC, et al. Antimicrobial susceptibility of Acinetobacter calcoaceticusAcinetobacter baumannii Complex and Stenotrophomonas maltophilia clinical isolates: results from the SENTRY antimicrobial surveillance program (1997-2016). Open Forum Infect Dis. 2019;6(Suppl 1):S34-46.

20. World Health Organization. AMR in the WHO European Region. 2019; Available from: http://www.euro.who.int/en/health-topics/diseaseprevention/antimicrobial-resistance/about-amr/amr-in-the-who-europeanregion. Cited 2019 May 21st.

21. Rosinski J, et al. Factors Shaping Attitudes of Medical Staff towards Acceptance of the Standard Precautions. Int J Environ Res Public Health. 2019;16(6):1050.

22. Różańska A, Wójkowska-Mach J, Bulanda M. Is the hospital environment friendly for infection control in Poland? Experience after twenty years of modern infection control. J Hosp Infect. 2016;94(3):228-9.

23. Weinshel $\mathrm{K}$, et al. Gap analysis of infection control practices in low- and middle-income countries. Infect Control Hosp Epidemiol. 2015;36(10):1208-14.

24. ECDC. Antimicrobial consumption: Annual epidemiological report for 2017. Stockholm: European Centre for Disease Prevention and Control; 2018.

25. Cao J, et al. Correlation between carbapenem consumption and antimicrobial resistance rates of Acinetobacter baumannii in a universityaffiliated hospital in China. J Clin Pharmacol. 2013:53(1):96-102.

26. Ogutlu A, et al. Effects of Carbapenem consumption on the prevalence of Acinetobacter infection in intensive care unit patients. Ann Clin Microbiol Antimicrob. 2014;13:7.

27. Yang $\mathrm{P}$, et al. Association between antibiotic consumption and the rate of carbapenem-resistant gram-negative bacteria from China based on 153 tertiary hospitals data in 2014. Antimicrob Resist Infect Control. 2018;7(1):137.

28. Goel N, et al. Trend analysis of antimicrobial consumption and development of resistance in non-fermenters in a tertiary care hospital in Delhi, India. J Antimicrob Chemother. 2011;66(7):1625-30.

29. Moreira MR, et al. Antimicrobial use, incidence, etiology and resistance patterns in bacteria causing ventilator-associated pneumonia in a clinicalsurgical intensive care unit. Rev Soc Bras Med Trop. 2013;46(1):39-44.

30. Fukuta $Y$, et al. Lack of seasonality in the occurrence of multidrug-resistant Acinectobacter baumannii complex. Infect Control Hosp Epidemiol. 2012; 33(10):1051-2.

31. Masiero $\mathrm{G}$, et al. Socioeconomic determinants of outpatient antibiotic use in Europe. Int J Public Health. 2010:55(5):469-78.

32. Koppe U, et al. Carbapenem non-susceptibility of Klebsiella pneumoniae isolates in hospitals from 2011 to 2016, data from the German antimicrobial resistance surveillance (ARS). Antimicrob Resist Infect Control. 2018;7:71

33. Adam HJ, et al. Comparison of pathogens and their antimicrobial resistance patterns in paediatric, adult and elderly patients in Canadian hospitals. J Antimicrob Chemother. 2013;68(Suppl 1):i31-7.

34. Schweickert B, et al. MRSA-surveillance in Germany: data from the antibiotic resistance surveillance system (ARS) and the mandatory surveillance of MRSA in blood. Eur J Clin Microbiol Infect Dis. 2012;31(8):1855-65.

35. Ayobami $\mathrm{O}$, et al. The Ongoing Challenge of Vancomycin-Resistant Enterococcus faecium and Enterococcus faecalis in Europe: An Epidemiological Analysis of Bloodstream Infections. Emerg Microbes Infect. 2020;9(1):1180-93.

36. Markwart $\mathrm{R}$, et al. The rise in vancomycin-resistant enterococcus faecium in Germany: data from the German antimicrobial resistance surveillance (ARS). Antimicrob Resist Infect Control. 2019:8(1):147.

37. Augustine $\mathrm{S}$, Bonomo RA. Taking stock of infections and antibiotic resistance in the elderly and long-term care facilities: a survey of existing and upcoming challenges. Eur J Microbiol Immunol (Bp). 2011;1(3):190-7.

38. Richter SE, et al. Risk Factors for Development of Carbapenem Resistance Among Gram-Negative Rods. Open Forum Infect Dis. 2019;6(3):ofz027.

39. Ayobami $\mathrm{O}$, et al. The incidence and prevalence of hospital-acquired (carbapenem-resistant) Acinetobacter baumannii in Europe, eastern Mediterranean and Africa: a systematic review and meta-analysis. Emerg Microbes Infect. 2019;8(1):1747-59.

40. Hafeez A, et al. ICU pathogens: a continuous challenge. J Coll Physicians Surg Pak. 2016;26(7):577-80.

41. Vincent $J$, et al. International study of the prevalence and outcomes of infection in intensive care units. JAMA. 2009;302(21):2323-9.

42. Kollef $M H$, Fraser $\mathrm{VJ}$. Antibiotic resistance in the intensive care unit. Ann Intern Med. 2001:134(4):298-314.

43. Villar $M$, et al. Epidemiologic and clinical impact of acinetobacter baumannii colonization and infection: a reappraisal. Medicine (United States). 2014. 93(5):202-10 
44. Uwingabiye J, et al. Intensive care unit-acquired Acinetobacter baumannii infections in a Moroccan teaching hospital: epidemiology, risk factors and outcome. GERMS. 2017;7(4):193-205.

45. ECDC. In: E.C.f.D.P.a. Control, editor. External quality assessment of laboratory performance - European Antimicrobial Resistance Surveillance Network (EARS-Net), 2017. Stockholm: European Centre for Disease Prevention and Control; 2018

\section{Publisher's Note}

Springer Nature remains neutral with regard to jurisdictional claims in published maps and institutional affiliations.

Ready to submit your research? Choose BMC and benefit from:

- fast, convenient online submission

- thorough peer review by experienced researchers in your field

- rapid publication on acceptance

- support for research data, including large and complex data types

- gold Open Access which fosters wider collaboration and increased citations

- maximum visibility for your research: over $100 \mathrm{M}$ website views per year

At BMC, research is always in progress. 